\title{
COMMENTARY
}

\section{The burden of adverse mental health outcomes in critical illness survivors}

\author{
Dimitry S Davydow* \\ See related research by Myhren et al., http://ccforum.com/content/14/1/R14
}

\begin{abstract}
Survivors of critical illnesses are at increased risk for posttraumatic stress disorder and major depression. In the present issue of Critical Care, Myhren and colleagues report on an investigation of predictors of posttraumatic stress, general anxiety, and depressive symptoms up to 1 year following intensive care unit admission for critical illnesses. The present study found that an endogenous patient characteristic, the personality trait pessimism, was associated with posttraumatic stress and depressive symptoms. Myhren and colleagues'study sheds light on potential predictors of psychopathology in critical illness survivors as well as stimulating directions for future research to address these complex problems.
\end{abstract}

Surviving a critical illness is inherently stressful, and survivors are frequently faced with complicated recoveries. Recently, there has been increased interest in the mental health of critical illness survivors. In the present issue of Critical Care, Myhren and colleagues report on the findings of a prospective investigation of posttraumatic stress, general anxiety, and depressive symptoms in 194 intensive care unit (ICU) survivors [1]. Their findings provide interesting and important insights into the development of psychopathology in critical illness survivors, which can serve to stimulate future investigations and inform clinical practice in the followup of these patients.

The point prevalence of symptoms suggestive of posttraumatic stress disorder (PTSD), as ascertained by an Impact of Events Scale cumulative score $\geq 35$, was $27 \%$ in this sample - slightly higher than the median point prevalence of symptoms suggestive of PTSD found in a

*Correspondence: ddavydo1@u.washington.edu

Department of Psychiatry and Behavioral Sciences, University of Washington

School of Medicine, 1959 NE Pacific Street, Box 356560, Seattle, WA 98195, USA recent systematic review of 15 studies of general ICU survivors [2], and within the range of clinically significant PTSD symptoms found in a systematic review of studies of acute lung injury survivors [3]. Furthermore, Myhren and colleagues identified a subpopulation whose level of PTSD symptoms actually increased over the 1-year follow-up period, a finding that highlights the need for ongoing follow-up of critical illness survivors.

Additionally, Myhren and colleagues found that a personality trait, pessimism (as ascertained by the Life Orientation Test), was an independent predictor of PTSD and depressive symptoms in their cohort of ICU survivors. This finding is noteworthy since it suggests that an endogenous patient-related factor, premorbid personality, may play an important role in conferring risk for post-ICU psychopathology. Personality traits such as neuroticism (a tendency towards negative emotions) are known to be a risk factor for the development of depressive and anxiety symptoms in the setting of stress $[4,5]$, suggesting that personality traits conferring a tendency towards a negative outlook may adversely affect the recovery of critical illness survivors.

It is important to note that this study does have limitations. First, Myhren and colleagues did not examine the role that a prior history of major depression and/or anxiety disorders (including PTSD) may have in increasing the risk of post-ICU psychopathology. Previous major depression and/or anxiety disorders are potent independent predictors of developing major depression and/or PTSD in the setting of stress [6,7], and a recent prospective investigation of traumatically injured ICU survivors found that pre-ICU depression was an independent predictor of symptoms suggestive of PTSD 1 year post discharge [8]. An additional limitation is that the Life Orientation Test was administered concurrently with the PTSD and depression measures, introducing the possibility that patients' current levels of PTSD and depressive symptoms affected their self-reports of personality traits.

Moreover, Myhren and colleagues do not report on important critical illness/ICU-related exposures that could confer risk for post-ICU psychopathology, such as 
medications received and ICU procedures. Benzodiazepine sedation has been found to be a potential risk factor for post-ICU PTSD [2], and a recent investigation of depressive symptoms in acute lung injury survivors found that benzodiazepine sedation predicted symptoms suggestive of major depression 6 months post discharge [9]. In addition, pulmonary artery catheterization was found to be an independent predictor of symptoms suggestive of the diagnosis of PTSD in traumatically injured ICU survivors 1 year after discharge [8]. Further research is needed to fully understand the role that these and other critical illness/ICU-related exposures could have in adversely affecting the mental health of ICU survivors.

Myhren and colleagues' study of PTSD, general anxiety and depressive symptoms up to 1 year after surviving an ICU admission for critical illness is an important contribution to the literature. This is only the second study to suggest that an endogenous patient-related characteristic, personality traits, is independently associated with PTSD and depressive symptoms after hospital discharge. This finding should stimulate further investigation into how other patient characteristics, such as possible genetic vulnerabilities and prior trauma exposure - an important predictor of PTSD in traumatic injury survivors [10] - influence the development of psychopathology in critical illness survivors. Since millions of patients are admitted annually to ICUs worldwide for the treatment of life-threatening critical illnesses, further research into the causes of post-ICU psychiatric morbidity is an important public health concern. Myhren and colleagues have taken an important step in the direction of furthering our understanding of the etiology of adverse mental health outcomes in ICU survivors as well as stimulating ideas for additional research into these complex problems.

\section{Abbreviations}

ICU, intensive care unit; PTSD, posttraumatic stress disorder.

\section{Acknowledgements}

The present work was supported by grant NRSA-T32/MH20021-11 from the National Institute of Mental Health.

\section{Competing interests}

The author declares that they have no competing interests.

Published: 25 February 2010

\section{References}

1. Myhren H, Ekeberg Ø, Tøien K, Karlsson S, Stokland O: Posttraumatic stress, anxiety and depression symptoms in patients during the first year post intensive care unit discharge. Crit Care 2010, 14:R14.

2. Davydow DS, Gifford JM, Desai SV, Needham DM, Bienvenu OJ: Posttraumatic stress disorder in general intensive care unit survivors: a systematic review. Gen Hosp Psychiatry 2008, 30:421-434.

3. Davydow DS, Desai SV, Needham DM, Bienvenu OJ: Psychiatric morbidity in survivors of the acute respiratory distress syndrome: a systematic review. Psychosom Med 2008, 70:512-519.

4. Kendler KS, Kuhn J, Prescott CA: The interrelationship of neuroticism, sex, and stressful life events in the prediction of episodes of major depression. Am J Psychiatry 2004, 161:631-636.

5. Bienvenu OJ, Stein MB: Personality and anxiety disorders: a review. J Personal Disord 2003, 17:139-151.

6. Kendler KS, Gardner CO, Prescott CA: Toward a comprehensive developmental model for major depression in men. Am J Psychiatry 2006, 163:115-124.

7. Brewin CR, Andrews B, Valentine JD: Meta-analysis of risk factors for posttraumatic stress disorder in trauma-exposed adults. J Consult Clin Psychol 2000, 68:748-766.

8. Davydow DS, Zatzick DF, Rivara FP, Jurkovich GJ, Wang J, Roy-Byrne PP, Katon WJ, Hough CL, Kross EK, Fan MY, Joesch J, MacKenzie EJ: Predictors of posttraumatic stress disorder and return to usual major activity in traumatically injured intensive care unit survivors. Gen Hosp Psychiatry 2009, 31:428-435.

9. Dowdy DW, Bienvenu OJ, Dinglas VD, Mendez-Tellez PA, Sevransky J, Shanholtz C, Needham DM: Are intensive care factors associated with depressive symptoms six months after acute lung injury? Crit Care Med 2009, 37:1702-1707.

10. Zatzick DF, Kang SM, Müller HG, Russo JE, Rivara FP, Katon W, Jurkovich GJ, Roy-Byrne P: Predicting posttraumatic distress in hospitalized trauma survivors with acute injuries. Am J Psychiatry 2002, 159:941-946.

doi:10.1186/cc8867

Cite this article as: Davydow DS: The burden of adverse mental health outcomes in critical illness survivors. Critical Care 2010, 14:125. 